\title{
Experience-Dependent Changes in Extracellular Spike Amplitude May Reflect Regulation of Dendritic Action Potential Back- Propagation in Rat Hippocampal Pyramidal Cells
}

\author{
Michael C. Quirk, Kenneth I. Blum, and Matthew A. Wilson \\ The Department of Brain and Cognitive Sciences, Center for Learning and Memory, and the RIKEN-Massachusetts \\ Institute of Technology Neuroscience Research Center, The Massachusetts Institute of Technology, Cambridge, \\ Massachusetts 02139
}

\begin{abstract}
Activity-dependent attenuations in extracellular spike amplitude have been shown to correlate with a decrease in the effectiveness with which somatic action potentials backpropagate into the dendritic arbor of hippocampal pyramidal cells. In this paper we demonstrate that activity-dependent attenuations in amplitude occur during behavior and that the amount of attenuation is reduced with an animal's experience in an environment. The observed reductions are caused by an animal's experience within a specific environmental context, are
\end{abstract}

dependent on functional NMDA receptors, and are accompanied by an increase in the effective coupling of pyramidal cells and interneurons. These results provide an important step in linking together in vivo studies with in vitro data and suggest that mechanisms of plasticity engaged during behavior may be sufficient to alter the biophysical and integrative properties of hippocampal pyramidal cells.

Key words: back-propagation; plasticity; hippocampus; freely behaving rat; NMDA; extracellular spike amplitude
The rodent hippocampus plays an essential role in an animal's ability to learn and remember spatial information (O'Keefe and Nadel, 1978). The hypothesis that the hippocampal formation is involved in the learning of spatial information is supported by the fact that hippocampal pyramidal cells fire within specific regions of space known as "place” fields (O'Keefe and Dostrovsky, 1971). When an animal enters the place field of a cell, the cell responds by producing both single spikes and bursts of action potentials known as complex spikes (Ranck, 1973). A common feature of these extracellularly recorded action potentials is an activitydependent attenuation in amplitude (Ranck, 1973; Quirk and Wilson, 1999). Combined intracellular and extracellular recordings in anesthetized rats have demonstrated that a decrease in extracellular spike amplitude correlates with an activitydependent decrease in the effectiveness with which somatic spikes actively back-propagate into the dendrites of hippocampal pyramidal cells (Buzsaki et al., 1996). In hippocampal slices, backpropagating action potentials are essential for certain forms of synaptic plasticity (Magee and Johnston, 1997), suggesting that mechanisms regulating back-propagation may play an important role in learning and memory. Although a number of factors, including synaptic input (Magee and Johnston, 1997), local inhibition (Tsubokawa and Ross, 1996), neuromodulation (Hoffman and Johnston, 1999; Sourdet and Debanne, 1999), and the recent activity of the neuron (Jaffe et al., 1992; Spruston et al., 1995), have been shown to influence the effectiveness with which spikes back-propagate into hippocampal dendrites in vitro, it remains to be determined how mechanisms regulating the active properties

Received June 27, 2000; revised Oct. 11, 2000; accepted Oct. 13, 2000.

We thank M. Mehta and A. Ogasawara for help with data collection. We also thank L. Frank, C. Lena, G. Liu, J. Levin, and T. McHugh for helpful discussions.

Correspondence should be addressed to Dr. Matthew A. Wilson, Department of Brain and Cognitive Sciences, Building E25-236, 45 Carleton Street, Cambridge, MA 02139. E-mail: wilson@ai.mit.edu.

Copyright (C) 2001 Society for Neuroscience 0270-6474/01/210240-09\$15.00/0 of hippocampal pyramidal cells are engaged within behaving animals. Because activity-dependent attenuations in the amplitude of extracellular spikes can serve as a signature of underlying intracellular changes (Henze et al., 2000), by monitoring changes in extracellular spike amplitude in awake and freely moving animals it is possible to relate changes in the biophysical properties of hippocampal pyramidal cells to an animal's behavior. In this paper, we demonstrate that the degree with which action potentials of hippocampal pyramidal cells show activitydependent attenuations in amplitude is reduced by an animal's experience in an environment. These changes are dependent on functional NMDA receptors, suggesting that one consequence of experience is to alter the biophysical and integrative properties of hippocampal pyramidal cells.

\section{MATERIALS AND METHODS}

Subjects and general procedures. Five 6- to 10-month-old male LongEvans rats (Charles River Laboratories, Wilmington, MA) served as subjects for these experiments. Animals were individually housed on a 12 $\mathrm{hr}$ light/dark cycle. Throughout the experiment, the rats were maintained at $85 \%$ of their free-feeding weights. Animals were trained to traverse continually between two food locations via restricted linear tracks (either a single linear track with food located at either end of the track and/or a $\mathrm{U}$-shaped track with food located at the two open ends of the U).

Surgical implantation and recording protocol. All animal care and surgical procedures were conducted in accordance with National Institutes of Health and Massachusetts Institute of Technology Animal Care guidelines. Briefly, each animal was anesthetized and chronically implanted with a microdrive array housing 12 independently adjustable tetrodes (Wilson and McNaughton, 1993; Quirk and Wilson, 1999). After surgery, animals were allowed to recover over a period of 7-10 d during which time the tetrodes were advanced into the pyramidal cell layer of the CA1 region of the dorsal hippocampus. Identification of the pyramidal cell layer was based on the depth of the tetrodes and on the occurrence of characteristic sharp wave and $200 \mathrm{~Hz}$ "ripple" activity. After stable pyramidal cells were isolated, experimental recordings were begun. An individual recording session consisted of a run period lasting between 8 and 30 min and bracketed by sleep periods of 10-60 min. During the sleep session, the animal rested on a small platform outside 
of the behavioral arena. During each recording session, both single-unit activity and the animal's position and head direction were monitored.

Neuronal signals and position information were sampled concurrently by the use of a series of eight synchronized 486DX-100 personal computers running custom-made acquisition software (AD, M. A. Wilson and L. Frank). For each channel within a tetrode, signals were bandpass filtered between $300 \mathrm{~Hz}$ and $6 \mathrm{kHz}$. Spike waveforms were amplified 10,000 times and sampled at $31.25 \mathrm{kHz}$ per channel. Position data were obtained by tracking a pair of infrared diode arrays mounted on a boom attached to the animal's head stage, such that one of the arrays was in front of the rat's nose and the other array was located above the animal's neck. Position data were sampled at a rate of $60 \mathrm{~Hz}$ with each diode array powered on alternate camera frames (allowing for the calculation of the animal's head direction). The tracking camera (Dragon Tracking) sampled a $256 \times 364$ pixel grid corresponding to a view of $156.3 \times 218.4 \mathrm{~cm}$. The intrinsic tracking error of the animal's position was $\sim 5 \mathrm{~cm}$. After each experimental session, data were transferred to a LINUX-based workstation for single-unit discrimination and further data analysis.

Unit isolation. Multiple single units recorded from a single tetrode were isolated by the use of computer software (XCLUST) developed by M. A. Wilson for the manual clustering of waveforms based on individual spike parameters. Because there is a slight spatial separation between the four wires that make up a tetrode, an important benefit of tetrode recordings is that spatially separated cells will produce spikes with different amplitudes but similar waveforms on each wire (channel). An additional benefit of tetrode recordings is that tetrodes are better suited for tracking systematic changes in spike shape than are single-wire electrodes (Wilson and McNaughton, 1993; Gray et al., 1995).

Isolated cells were classified as either excitatory pyramidal cells or interneurons on the basis of their specific firing characteristics. Pyramidal cells were those cells that displayed incidents of complex spike bursts and had broad waveforms. Interneurons had relatively narrow waveforms and showed a complete absence of complex spikes (Ranck, 1973; Csicsvari et al., 1998, 1999). Only cells that fired a minimum of 100 spikes during a run session were analyzed.

\section{RESULTS}

Previous studies (Ranck, 1973; Quirk and Wilson, 1999) have demonstrated that extracellularly recorded action potentials from hippocampal pyramidal cells show a dramatic and activitydependent attenuation in amplitude during behavior (Fig. 1). Although these attenuations in amplitude occur over multiple timescales, they are most dramatic during high-frequency bursts (Ranck, 1973; Quirk and Wilson, 1999). To determine whether an animal's experience within an environment alters these attenuations in amplitude, a total of 152 hippocampal pyramidal cells with place-specific activity were recorded from five male LongEvans rats as each rat ran for food reward in a variety of familiar environments. Bursts of spikes (interspike interval $<10 \mathrm{msec}$ ) were isolated from the spike trains of each pyramidal cell active within an environment. For each burst event, the total amount of attenuation within a burst was determined by dividing the amplitude of the last spike in the burst by the amplitude of the first spike. Because the total amount of attenuation within a burst is dependent on the number of spikes within a burst as well as the interval between spikes (Quirk and Wilson, 1999), experiencedependent comparisons were made only between bursts consisting of the same number of spikes.

Figure $2 A$ shows, for a population of simultaneously recorded cells $(n=28)$, the average amplitude attenuation within bursts for both the first $4 \mathrm{~min}$ and the last $4 \mathrm{~min}$ of an animal's experience within an environment. For this recording session there was a significant (Fig. 2A; ${ }^{*} p<0.05$, paired $t$ test) decrease in amplitude attenuation as a consequence of experience. That is, later bursts exhibited less attenuation in amplitude than did earlier bursts.

A total of seven data sets were analyzed from the five animals. Because some recording sessions contained few bursts with more
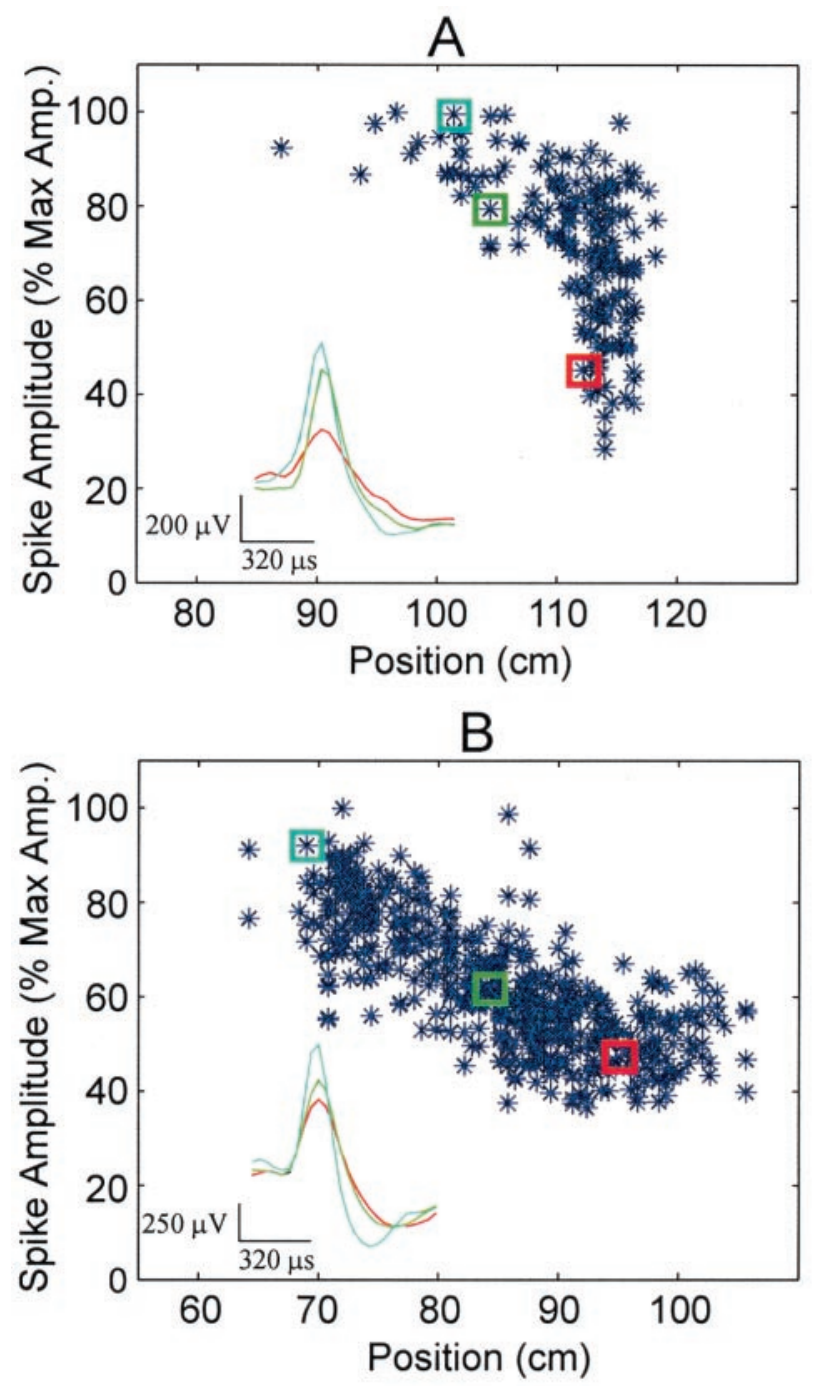

Figure 1. The amplitude of extracellularly recorded spikes decreases as an animal runs through the place field of a cell. $A, B$, Amplitude of individual spikes, from two representative hippocampal pyramidal cells, plotted as a function of the rat's location on a track. The cells were directionally tuned and fired as the animal moved from left to right on the $x$-axis. The amplitude of each spike is expressed as a percentage of the maximum spike amplitude recorded from the cell. Insets, The waveforms of three spikes recorded from a single-tetrode channel. Because a systematic decrease in amplitude was seen simultaneously on all four channels of the tetrode, the spikes are assumed to have originated from the cell. Colored boxes outline those spikes whose waveforms are depicted in the insets. Amp, Amplitude; Max, maximum.

than three spikes, the analysis of changes in spike amplitude attenuation was based on differences between bursts consisting of no more than three spikes. Figure $2 B$ shows, for each of the seven recording sessions, the average change in amplitude for bursts of three spikes for both the first $4 \mathrm{~min}$ and the last $4 \mathrm{~min}$ of an animal's experience within an environment. All seven of the recording sessions ( $p<0.05$, sign test) showed significant reductions in amplitude attenuation as a consequence of experience. For these seven data sets, results for bursts of more than three spikes were generally consistent with that for three-spike bursts but were more variable because of a lower incidence of occurrence and a floor effect caused by noise and cell classification thresholds (Buzsaki et al., 1996). 

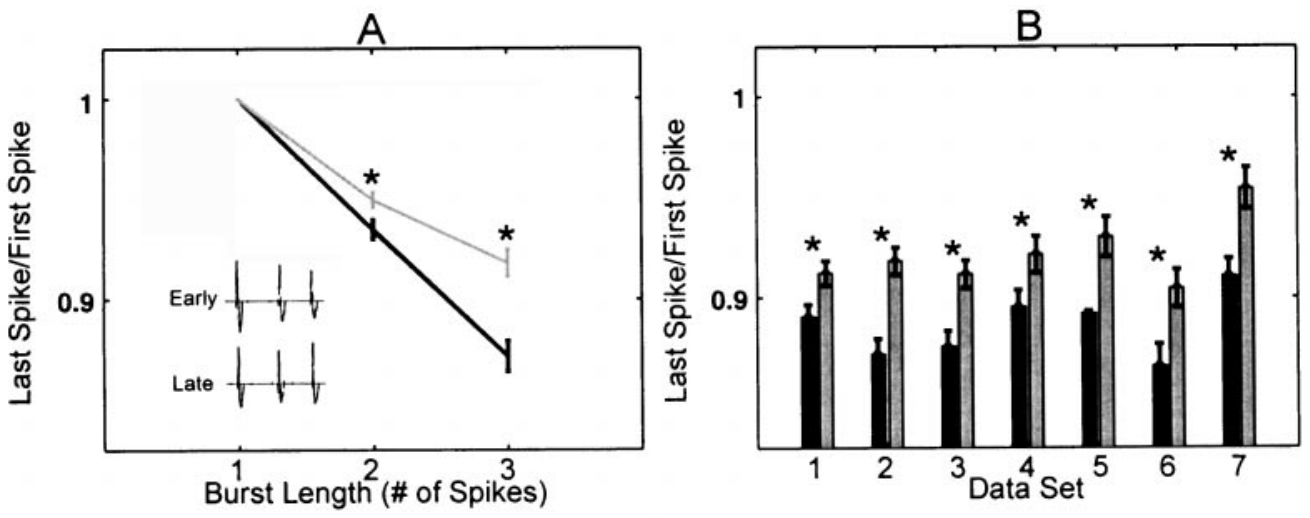

Figure 2. Activity-dependent attenuation in spike amplitude is reduced with experience. $A$, The average $( \pm$ SE) amplitude attenuation during high-frequency bursts for a population of simultaneously recorded cells. The amplitude of the last spike in a burst is expressed as a fraction of the amplitude of the first spike and is plotted as a function of the number of spikes in the burst. The black line plots the average attenuation for the animal's first $4 \mathrm{~min}$ in the environment, and the gray line plots the attenuation for the animal's last 4 min. Notice that the amount of attenuation is reduced with experience (an asterisk indicates a significant difference, $p<0.05$, paired $t$ test). $B$, The average attenuation for bursts of three spikes for both the first 4 min (black bars) and last 4 min ( gray bars) of an animal's experience in a familiar environment. A significant $\left({ }^{*} p<0.05, t\right.$ test) reduction in amplitude attenuation was seen in seven of the seven data sets.

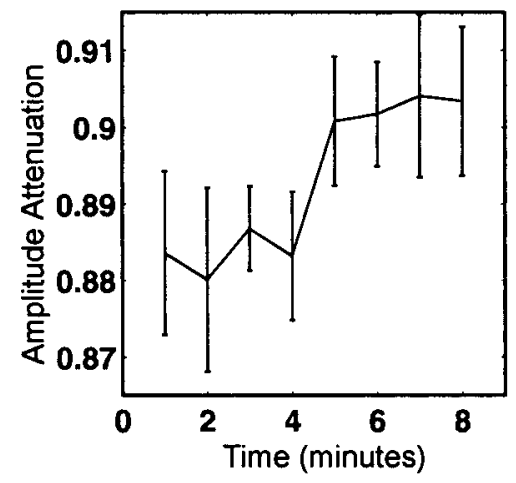

Figure 3. Time course of changes in amplitude attenuation during behavior. Average $( \pm \mathrm{SE})$ amplitude attenuation within bursts of three spikes as a function of the time spent within an environment.

\section{Time course of changes in amplitude attenuation during behavior}

Previous studies have demonstrated that the place fields of CA1 pyramidal cells undergo dramatic changes in size, shape, and location as a consequence of an animal's experience within both novel and familiar environments (Wilson and McNaughton, 1993; Mehta et al., 1997, 2000). A common feature of these changes is that they occur very rapidly within the first few minutes of an animal's active exploration of an environment. Although our initial characterization of experience-dependent changes in amplitude attenuation compared the average amplitude attenuation during an animal's first 4 min in an environment with the average amount of attenuation observed during an animal's last $4 \mathrm{~min}$ within an environment, Figure 3 shows the average amount of amplitude attenuation within bursts of three spikes as a function of time. Because of the minute-by-minute variability in the number of spike events within any given data set, data were pooled across all data sets. As can clearly be seen from Figure 3, the magnitude of amplitude attenuation remained relatively constant during the animal's first few minutes within an environment but showed a dramatic change in average attenuation after the animal had explored an environment for $\sim 4 \mathrm{~min}$. Thus, the time course over which bursts of spikes from hippocampal pyramidal cells show an experience-dependent reduction in amplitude attenuation is consistent with previously reported changes in the activity of place cells (Mehta et al., 2000).

\section{Reductions in amplitude attenuation are not caused by a change in interspike interval or a change in first-spike amplitude}

Amplitude attenuation within bursts is frequency dependent (Ranck, 1973; Buzsaki et al., 1996); as a consequence, an experience-dependent increase in the interval between spikes within a burst would lead to a reduction in amplitude attenuation. However, in only one of the seven data sets was there a significant $(p<0.05, t$ test) increase in the average interspike interval (ISI) distribution. Conversely, one data set showed a significant decrease in average ISI within bursts. These results suggest that the observed changes in amplitude attenuation were therefore not caused by an increase in the interval between spikes within a burst. Furthermore, none of the data sets showed a significant change in the average first-spike amplitude between the first 4 min and the last 4 min of a recording session. Thus, the observed changes in attenuation are not easily explained as being a result of the normalization process or of nonspecific effects that altered spike amplitude in general [e.g., temperature (Andersen and Moser, 1995)].

\section{Reductions in amplitude attenuation are environmentally specific}

One potential mechanism that could be responsible for the reduction in amplitude attenuation is a nonspecific change in cellular excitability that is caused simply by the fact that when the animal is introduced into an environment the animal goes from a resting state to a state in which it is highly active. Alternatively the modifications underlying the reduction in attenuation could be caused by the animal's experience within a specific environmental context. If the observed changes in attenuation were specific to an animal's experience within a particular environment, changing the environment in which the animal ran should cause the reductions in amplitude attenuation to return to baseline values. To test this hypothesis, two of the five animals were recorded on one familiar track and then transferred immediately to another familiar track. In agreement with previous experi- 

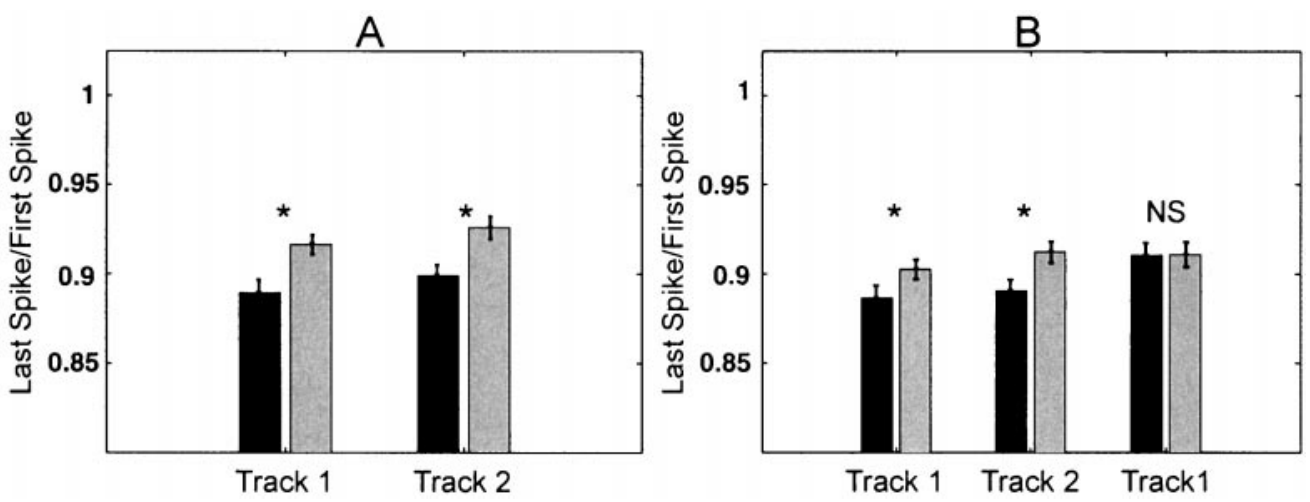

Figure 4. Experience-dependent reductions in amplitude attenuation are context specific. $A$, Two animals were allowed to run on one familiar track and then immediately transferred to a second familiar track. A subset of hippocampal cells $(n=48)$ was active on both tracks. For these cells, the average amplitude attenuation for bursts of three spikes is shown. The black bars show the average attenuation for the animal's first $4 \mathrm{~min}$ in the environment, and the gray bars show the average attenuation for the animal's last $4 \mathrm{~min}$. Notice that in the first environment (track 1), bursts showed an experiencedependent reduction in amplitude attenuation $\left({ }^{*} p<0.05, t\right.$ test $)$. When the animal was transferred to the second environment (track 2), the amount of attenuation initially increased relative to that in the last 4 min in the first environment but was once again reduced with experience ( ${ }^{*} p<0.05, t$ test). $B$, To determine whether handling alone was sufficient to cause reductions in amplitude attenuation to reset when an animal was transferred from one track to another, one animal was returned to track 1 after experience on track 2 . For this animal, a total of 21 hippocampal cells were active on both tracks. For these cells, the average amplitude attenuation for bursts of three spikes is shown. The black bars show the average attenuation for the animal's first 4 min in the environment, and the gray bars show the average attenuation for the animal's last 4 min. When the animal was returned to the first environment (track 1), the amount of attenuation was the same as when the animal left this environment, suggesting that handling alone does not cause the amount of attenuation to return to baseline.

ments (Mehta et al., 1997, 2000), a subset of pyramidal cells was active on both tracks $(n=48)$. For those cells active in both environments, we calculated the average amplitude attenuation within each environment. In agreement with our previous results, there was a decrease in amplitude attenuation as a function of the animal's experience on track 1 (Fig. $4 A$; average fractional amplitude of last spike during first $4 \mathrm{~min}=0.8895$; average fractional amplitude of last spike during last $4 \mathrm{~min}=0.9165 ;{ }^{*} p<0.05, t$ test, Bonferroni corrected for multiple $t$ tests). When the animal was transferred to the second track, the amount of amplitude attenuation for the first $4 \mathrm{~min}$ on track 2 (average fractional amplitude of last spike $=0.8991, \mathrm{SD}=0.1196$ ) was significantly greater than the average amplitude attenuation for the last $4 \mathrm{~min}$ on track 1 (average fractional amplitude of last spike $=0.9165$, $\mathrm{SD}=0.1384 ; p<0.05, t$ test, Bonferroni corrected for multiple $t$ tests) but was not significantly different from the initial amplitude attenuation on track 1 (average fractional amplitude of last spikes $=0.8895, \mathrm{SD}=0.1346 ; p>0.05, t$ test, Bonferroni corrected for multiple $t$ tests). However, with experience, the amplitude attenuation on track 2 once again decreased (average fractional amplitude of last spike $=0.9261, \mathrm{SD}=0.1372 ;{ }^{*} p<$ 0.05 , Bonferroni corrected for multiple $t$ tests). Thus, a change in environment led to a resetting of the average amplitude attenuation within bursts.

Although the rat was only handled briefly (1-5 sec) while being transferred from track 1 to track 2 , this handling could have been responsible for the observed resetting in the degree of amplitude attenuation. To exclude this possibility, one animal was returned to track 1 after experience on the second track. For this animal a total of 21 cells were active on both track 1 and track 2 . As Figure $4 B$ shows, when the animal was returned to the first track, the average amplitude attenuation remained decreased and was not significantly different from that when the animal left track $1(p>$ $0.05, t$ test, Bonferroni corrected for multiple $t$ tests). Thus, the fact that the amplitude attenuation reset when the animal was initially moved from track 1 to track 2 was not simply a result of handling the animal. These results suggest that changes in spike amplitude attenuation are indeed dependent on an animal's experience within a specific environmental context and are not solely caused by a nonspecific change in cellular excitability.

\section{Reductions in amplitude attenuation are not correlated with a decrease in the firing of inhibitory interneurons}

In vitro, shunting inhibition, caused by the activation of hippocampal interneurons, can cause a reduction in the amplitude of back-propagating action potentials (Tsubokawa and Ross, 1996). Similarly, Buzsaki et al. (1996) have shown that an increase in the amount of inhibitory input onto a pyramidal cell can cause an increase in the degree to which extracellularly recorded spikes show activity-dependent attenuations in amplitude. The observation that activity-dependent attenuations in spike amplitude are reduced with an animal's experience in an environment may therefore be caused by an experience-dependent reduction in inhibition. One potential indicator of whether the amount of hippocampal inhibition changes with experience is a change in the firing rate of putative interneurons (Wilson and McNaughton, 1993). Thus, it is important to determine how the firing of interneurons within the hippocampus relates to changes in amplitude attenuation. Eleven times during our experiments, a single tetrode recorded from both a putative inhibitory interneuron and a population of pyramidal cells. For each such tetrode we determined the average change in amplitude attenuation for all pyramidal cells on the tetrode. We also determined the change in the firing rate of each interneuron for both the first $4 \mathrm{~min}$ and the last $4 \mathrm{~min}$ of the animal's experience in an environment. If one would expect reductions in amplitude attenuation to be correlated negatively with changes in interneuron firing rate. In fact, a positive but nonsignificant correlation was found between reductions in attenuation and changes in interneuron firing rate $(r=0.49 ; p>0.05)$. Thus, a decrease in interneuron firing rate was not a necessary condition for pyramidal cells to show experience-dependent reductions in amplitude attenuation.

\section{Experience-dependent changes in the effective connectivity of pyramidal cells and interneurons}

Changes in the amount of inhibition onto pyramidal cells may be mediated by changes in the strength of coupling between excitatory and inhibitory neurons that are not reflected in changes in the overall interneuron firing rate (Grunze et al., 1996). In particular, it has been suggested that short-latency peaks $(2-3 \mathrm{msec})$ reductions in attenuation were caused by a decrease in inhibition, 
Figure 5. The effective connectivity between hippocampal pyramidal cells and interneurons is increased with an animal's experience in an environment. Normalized crosscorrelation histograms were computed for pairs of pyramidal cells and interneurons recorded from the same tetrode during behavior (see Results). $A, B$, Normalized crosscorrelation histograms for one pyramidal cell-interneuron pair for both the animal's first $4 \min (A)$ and last $4 \min (B)$ in an environment. The short-latency $(2-3 \mathrm{msec})$ peak in the cross-correlation histogram represents a putative monosynaptic connection between the pyramidal cell and interneuron (dotted line at $3 \mathrm{SD}$ above mean, $p<0.0013$ ). For this cell pair, the magnitude of the monosynaptic peak increases with experience, suggesting an experience-dependent increase in effective coupling. For these experiments, 9 of 12 "coupled" pyramidal cell-interneuron pairs showed an experience-dependent increase in effective connectivity $(p<0.05$, sign test).

in the cross-correlation of hippocampal interneurons with respect to the firing of pyramidal cells reflect monosynaptic connections onto interneurons within the pyramidal cell layer (Csicsvari et al., 1998). These interneurons are believed to provide feedback inhibition onto the pyramidal cells throughout the hippocampus and are therefore in an ideal position to regulate the excitability of the proximal dendrites of pyramidal cells. Because a reduction in pyramidal cell-interneuron coupling could reduce feedback inhibition and in turn reduce attenuations in spike amplitude, we were interested in determining whether the effective coupling between pyramidal cells and interneurons changed with an animal's experience within an environment.

To examine changes in pyramidal cell-interneuron coupling, cross-correlations were computed between those pyramidal cells and interneurons that were recorded from the same tetrode (Csicsvari et al., 1998). Each pyramidal cell-interneuron crosscorrelation histogram was centered on the occurrence of a pyramidal cell spike and was normalized by the firing rate of the pyramidal cell. Cross-correlations were computed separately for both the first $4 \mathrm{~min}$ and the last $4 \mathrm{~min}$ of an animal's experience within an environment. To control for changes in baseline correlation, the average value for all bins within $\pm 100 \mathrm{msec}$ was subtracted from the peak value at short latency $(2-3 \mathrm{msec})$. In addition, because neurons within the hippocampus are rhythmically modulated during behavior, which could in turn produce a broad peak in the cross-correlation, for each cross-correlation we calculated a local mean based on the cross-correlation $20 \mathrm{msec}$ before and after the peak bin. Cells were determined to be "coupled" if, in at least one of the two time epochs, there was a significant ( $>3$ SD above the local mean; $p<0.0013$ ) peak in the cross-correlation histogram 2-3 msec after the occurrence of a pyramidal cell spike. On the basis of these criteria, a total of 12 pyramidal cell-interneuron pairs were classified as being coupled. Figure 5 shows two cross-correlation histograms for one such pyramidal cell-interneuron pair. These two histograms reflect the effective coupling between these two neurons for both the first and last 4 min of an animal's experience within an environment and demonstrate that the coupling between these two neurons increased as a function of the animal's experience within the environment. Of the 12 effectively coupled neuron pairs, 9 pairs showed an increase in effective coupling between the first and last 4 min of an animal's experience in an environment $(p<0.05$, sign test). These results suggest that reductions in amplitude attenuation are not caused by a decrease in pyramidal cellinterneuron coupling. Rather, reductions in amplitude attenuation within pyramidal cells are accompanied by an increase in the effective coupling of pyramidal cells and interneurons during behavior.

\section{Inhibition of the NMDA receptor alters activity- dependent attenuations in spike amplitude}

The activation and inactivation properties of dendritic potassium and sodium channels govern effective back-propagation in vitro (Colbert et al., 1997; Hoffman et al., 1997; Jung et al., 1997). In particular, the activation of A-type potassium channels limits the spread of action potentials within dendrites, and the kinetics of sodium channel inactivation helps to determine the magnitude of amplitude attenuation during trains of spikes. In hippocampal slices, modest levels of dendritic depolarization have been shown to increase the spread of back-propagating action potentials by reducing the pool of available potassium channels (Hoffman et al., 1997). Thus, by increasing the tendency for A-type channels to be in an inactivated state, an increase in dendritic depolarization might lead to a reduction in amplitude attenuation during behavior. Furthermore, modulation of the (in)activation properties of both dendritic sodium and potassium channels via protein phosphorylation has been shown to increase effective backpropagation in vitro (Hoffman and Johnston, 1998). Because an enhancement in synaptic strength can lead to both an increase in dendritic depolarization and the activation of a variety of protein kinase pathways (for review, see Roberson et al., 1996), experience-dependent reductions in amplitude attenuation may be caused by an experience-dependent increase in synaptic strength. Because experience-dependent increases in synaptic strength are thought to require functional NMDA receptors (Morris et al., 1986; Bear and Malenka, 1994; Mehta et al., 2000), inhibitors of the NMDA receptor should alter the attenuation characteristics of hippocampal pyramidal cells. To test this prediction, three animals were injected intraperitoneally with $3-[(R)-$ 2-carboxypiperazin-4-yl] (CPP; 5-10 mg/kg), a potent NMDA receptor inhibitor. Injections of $10 \mathrm{mg} / \mathrm{kg}$ CPP have been shown previously to block the induction of long-term potentiation (LTP) in vivo and have also been shown to affect the long-term stability of hippocampal place cells (Kentros et al., 1998). A total of eight data sessions were analyzed from the three animals (Fig. 6). Although there were no overt behavioral differences between 
A

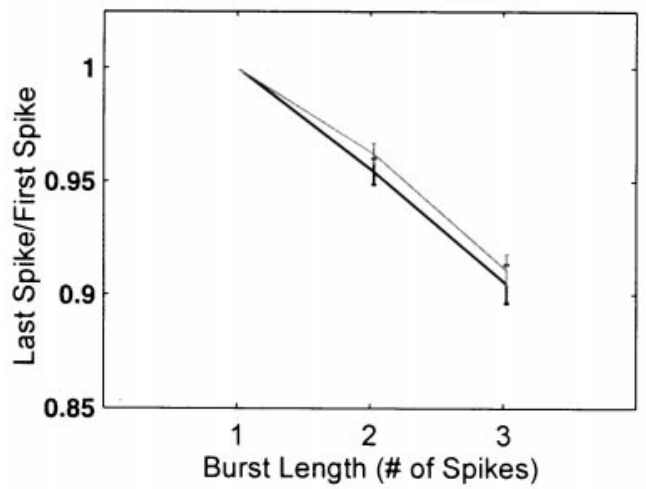

B

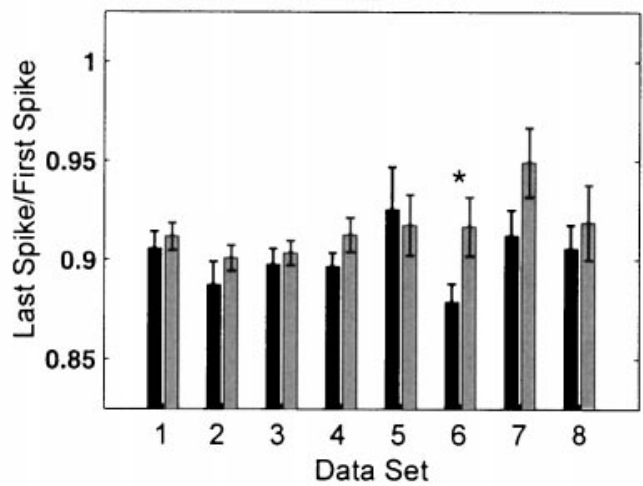

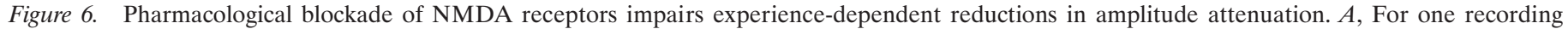

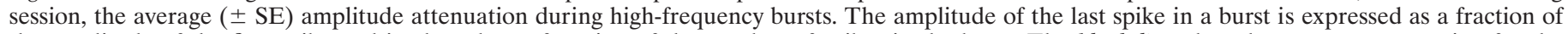

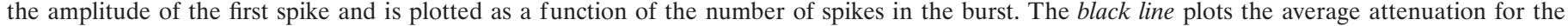

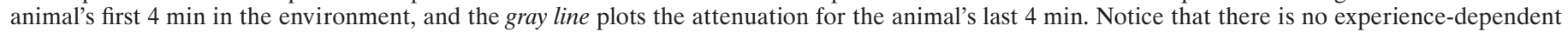

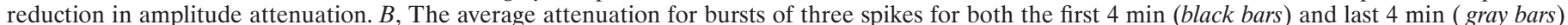

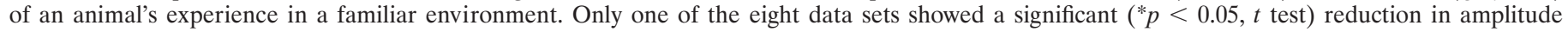
attenuation.

CPP and control animals, there were significant differences in the amplitude attenuation properties of cells recorded from CPP animals compared with those recorded from control animals.

First, in comparison with control animals, CPP animals showed, on average, slightly less amplitude attenuation in the first 4 min of an animal's experience in an environment $(p<0.05$, rank order sum). A difference in baseline attenuation between CPP and control animals is consistent with the observation that, in hippocampal slices, blockade of NMDA receptors alters local circuit inhibition (Grunze et al., 1996). In particular, NMDA antagonists reduce the size of IPSPs caused by feedback inhibition onto pyramidal cells. By reducing shunting currents, a reduction in feedback inhibition would reduce the amount of amplitude attenuation within hippocampal pyramidal cells. Consistent with this hypothesis, a post hoc analysis of two interneurons recorded across several CPP (four sessions) and control (three sessions) conditions showed a significant reduction in firing rate during the CPP sessions ( $p<0.05$, one-tailed $t$ test). This suggests that the difference in baseline attenuation between control and CPP animals may be caused by a reduction of feedback inhibition within CPP-injected animals.

The second difference between CPP and control animals is that seven of eight data sets from the CPP animals showed no significant change in amplitude attenuation as a consequence of an animal's experience in an environment. The one data set that showed a significant experience-dependent reduction received the smallest dosage of CPP (5 mg/kg) (Fig. 6B, data set 6). On a subsequent day, the same animal was injected with a higher dosage of CPP $(10 \mathrm{mg} / \mathrm{kg})$, and on this day, there was no experience-dependent reduction in amplitude attenuation. Thus, the observed reduction in the previous data set was probably caused by an incomplete blockade of the NMDA receptors. Finally, a comparison of the average experience-dependent change in attenuation for both control data sets (mean $=0.0356$; $\mathrm{SD}=0.0089 ; n=7)$ and CPP data sets (mean $=0.0140 ; \mathrm{SD}=$ $0.0141 ; n=8$ ) also revealed a significant difference between CPP and control animals $(p=0.0042)$. On the basis of these results, we conclude that the effective blockade of NMDA receptors, although not eliminating changes in amplitude attenuation, significantly reduces the magnitude by which spikes from hippocam- pal pyramidal cells show experience-dependent reductions in attenuation.

\section{DISCUSSION}

In hippocampal slices, back-propagating action potentials are essential for certain forms of synaptic plasticity (Magee and Johnston, 1997), suggesting that mechanisms regulating backpropagation may play an important role in learning and memory. Although recent two-photon imaging studies in anesthetized rats have directly demonstrated that somatic action potentials are capable of actively back-propagating into the dendrites of certain cortical neurons in vivo (Helmchen et al., 1999), little is known about the factors regulating effective back-propagation within freely behaving animals. Previously, it has been estimated that tetrodes isolate signals from a spherical volume of tissue with a diameter of $\sim 130 \mu \mathrm{m}$ (Gray et al., 1995). A single tetrode is therefore able to record action potentials not only from a population of cells but also from both the soma and proximal dendrites of any given hippocampal cell within the recording area. Because extracellularly recorded action potentials are bound to reflect an integrated signal derived from both a pyramidal cell's soma and proximal dendrites (Buzsaki et al., 1996), changes in the active and passive properties of either of these compartments will alter the amplitude of extracellular spikes (Fig. 7). Activity-dependent changes in the amplitude of extracellularly recorded action potentials can therefore serve as a signature of underlying changes in intracellular processing. Furthermore, because of differences in the kinetics of somatic versus dendritic ion channels (Colbert et al., 1997; Jung et al., 1997), during repetitive firing, dendritic action potentials will attenuate more quickly than will somatic spikes (Spruston et al., 1995). As a consequence changes in extracellular spike amplitude within bursts of spikes are likely to reflect changes in the effectiveness with which spikes are generated within a cell's dendrites (Buzsaki et al., 1996). Here, we have shown that the degree to which the action potentials of hippocampal pyramidal cells show activity-dependent reductions in amplitude is reduced by an animal's experience within an environment. Furthermore, the observed reductions are dependent on functional NMDA receptors, suggesting that mechanisms of plasticity 


\section{Early}

First Spike

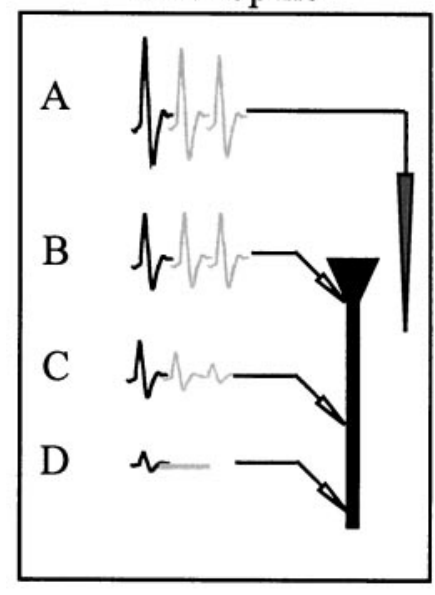

First Spike

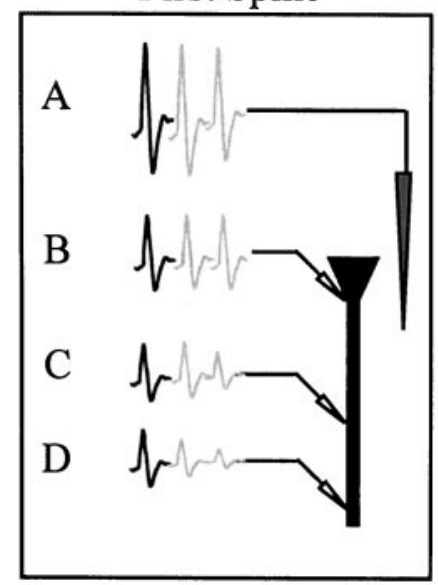

Second Spike

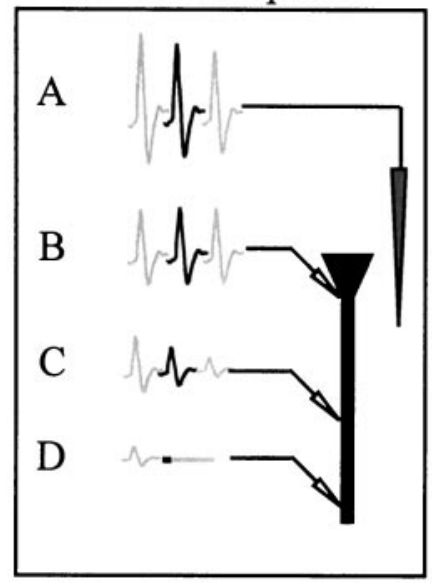

Late

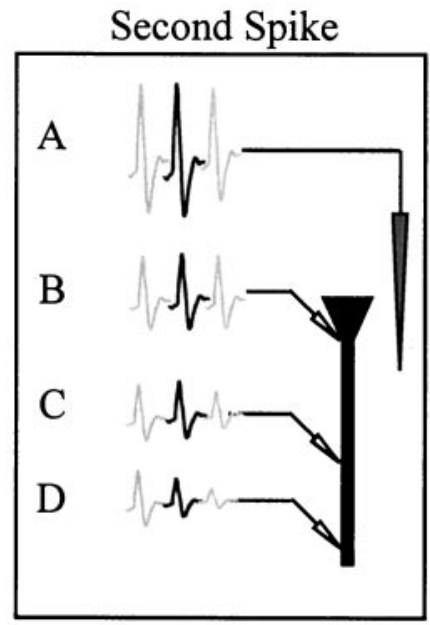

Third Spike

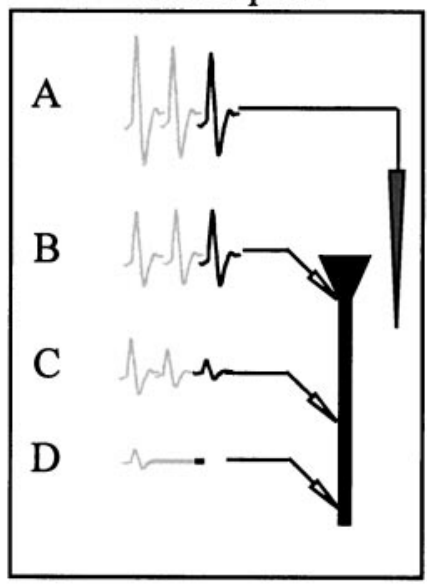

Third Spike

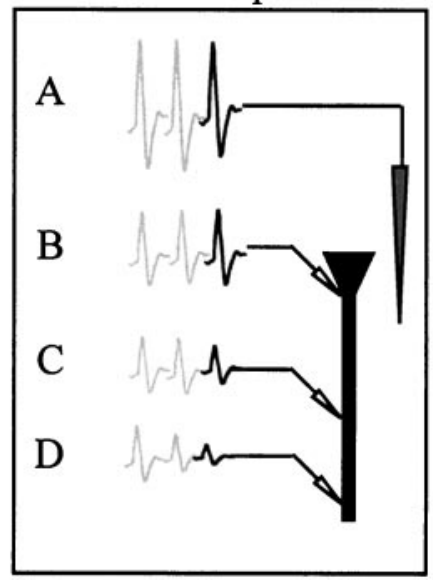

Figure 7. Extracellular electrodes record from a local volume of tissue, and as a consequence an extracellular action potential may reflect an integrated signal from both the soma and proximal dendrites of a hippocampal pyramidal cell. Top, During a burst of action potentials there is an activity-dependent decrease in the ability of later spikes within a burst to back-propagate actively into the cell's dendrites. This activity-dependent decrease in effective back-propagation may manifest as a decrease in the amplitude of extracellularly recorded spikes $(A$, extracellularly recorded signal; $B$, intracellular somatic signal; $C$, intracellular signal from proximal dendrites; $D$, intracellular signal from distal dendrites). Bottom, An experience-dependent reduction in the degree to which extracellularly recorded action potentials show activity-dependent attenuations in spike amplitude may reflect an increase in the effectiveness with which trains of intracellular action potentials actively back-propagate into the dendrites of hippocampal pyramidal cells $(A-D$, same as in the top panels).

engaged during behavior may be sufficient to alter the biophysical properties of hippocampal pyramidal cells.

\section{Mechanisms regulating activity-dependent attenuations in spike amplitude}

How might an animal's experience within an environment lead to reductions in amplitude attenuation? In hippocampal slices, one factor regulating the spread of action potentials within the dendritic arbor of a pyramidal cell is the strength of inhibitory input onto the cell. Specifically, during a train of action potentials, feedback inhibition can produce a shunting current that will reduce the amplitude of spikes within the train (Tsubokawa and Ross, 1996). Two potential signatures of changes in hippocampal inhibition are (1) changes in the firing rate of interneurons (Wilson and McNaughton, 1993) and (2) changes in the effective coupling between pyramidal cells and interneurons (Csicsvari et al., 1998). Using both of these measures, we found no significant correlation between experience-dependent reductions in amplitude attenuation and a decrease in inhibition. Rather, there was a tendency for the coupling between pyramidal cells and interneurons to increase with an animal's experience within an environment. Thus, our results suggest that reductions in hippocampal inhibition are not responsible for experience-dependent changes in the magnitude of amplitude attenuation within a pyramidal cell.

Another factor regulating the spread of action potentials within the dendrites of hippocampal pyramidal cells in vitro is the activation kinetics of A-type potassium channels (Hoffman et al., 1997). Both experimental and modeling data have indicated that 
modest levels of dendritic depolarization can decrease the population of available A-type channels and thereby increase the size of dendritic action potentials (Hoffman et al., 1997). Furthermore, subthreshold synaptic activity has been shown to regulate the size of back-propagating action potentials in hippocampal slices (Magee and Johnston, 1997), suggesting that large EPSPs may be sufficient to inactivate temporarily A-type potassium channels. Thus, one mechanism for reductions in amplitude attenuation is that as an animal becomes familiar with an environment, inputs onto an active cell become potentiated (Mehta et al., 2000), in turn increasing the tendency for A-type channels to be in an inactivated state and thereby increasing the effectiveness with which action potentials propagate within the dendritic arbor the cell. Because NMDA receptors are believed to be important for synaptic changes with CA1 in behaving animals (McHugh et al., 1996), this explanation is consistent with the observation that NMDA antagonists block experience-dependent reductions in amplitude attenuation. Furthermore, because it is presumed that in a second environment cells are initially being driven by unpotentiated inputs, this explanation is also consistent with the observation that the magnitude of amplitude attenuation within a cell resets when the animal is switched to a different environment. A specific prediction of this hypothesis is that induction of LTP within a slice should increase the effectiveness with which subsequent trains of spikes back-propagate. Recent in vitro results demonstrate that strong depolarizing pulses to hippocampal dendrites can lead to a long-term reduction in the attenuation of subsequent trains of back-propagating action potentials (Tsubokawa et al., 2000), supporting the hypothesis that previous electrical stimulation is sufficient to alter effective backpropagation within hippocampal dendrites.

Finally, experience-dependent reductions in amplitude attenuation may be caused by an experience-dependent change in the level of neuromodulators within the hippocampus. In particular, it is known that, in hippocampal slices, cholinergic agonists reduce activity-dependent attenuations in dendritic spike amplitude (Tsubokawa and Ross, 1997) by altering the inactivation kinetics of dendritic sodium channels (Colbert et al., 1997). Microdialysis experiments, in vivo, have demonstrated that levels of acetylcholine (ACh) within the hippocampus can be influenced by an animal's relative familiarity with an environment (Sarter and Bruno, 1997). Thus, experience-dependent changes in spike amplitude attenuation may reflect a change in neuromodulatory input to the hippocampus that is dependent on an animal's relative familiarity with an environment. Although the CPP data would suggest that NMDA receptors play an important role in modifying experience-dependent changes in spike amplitude attenuation, it must be stressed that systemic CPP injections are also known to alter ACh levels within the brain (Giovannini et al., 1997). Thus, more specific pharmacological and or genetic manipulations are necessary to determine the exact mechanism responsible for changes in spike amplitude attenuation in vivo.

\section{Functional role for modulation of the active properties of dendrites over different timescales during behavior}

Changes in the amplitude of extracellularly recorded action potentials can be broadly divided into two categories: (1) attenuations in spike amplitude that occur during a single train of spikes and (2) changes in the magnitude of attenuation that occur as a consequence of an animal's experience within an environment. Whereas rapid changes in amplitude within a train may be related to the activation and inactivation properties of a cell's ion chan- nels and changes in the membrane potential of the cell (Henze et al., 2000), experience-dependent changes in the magnitude of attenuation suggest that mechanisms of plasticity engaged during behavior may alter the biophysical properties of hippocampal pyramidal cells. Because voltage-gated ion channels within hippocampal dendrites are known to alter both the integration and transmission of electrical signals within a pyramidal cell, in vitro (Magee and Johnston, 1995; Johnston et al., 1996; Hoffman et al., 1997; Sourdet and Debanne, 1999), what may be the functional implications of these changes in extracellular spike amplitude during behavior?

\section{Attenuations in spike amplitude during a single pass through a place field}

As reported previously, because of rapid activity-dependent attenuations in spike amplitude, action potentials occurring as the animal enters a cell's place field will be of greater amplitude than will those produced as the animal exits the cell's field (Quirk and Wilson, 1999), suggesting that they will propagate more effectively into the cell's dendritic arbor than will later spikes. In vitro calcium-imaging studies have demonstrated that, in hippocampal pyramidal cells, the amount of calcium entering a dendritic compartment may depend on the spread of sodium action potentials (Jaffe et al., 1992; Magee and Johnston, 1997). Because calcium levels may help to determine the direction and degree of synaptic modification (Yang et al., 1999), inputs that arrive early in a train of action potentials produced by a postsynaptic cell (i.e., initial region of the cell's place field) are more likely to be potentiated than are later inputs. Activity-dependent attenuations in spike amplitude may therefore serve as a mechanism for asymmetrically strengthening the inputs onto a cell over behaviorally relevant timescales of hundreds of millisecond to seconds. Asymmetric synaptic plasticity is theoretically important for both sequence learning and rodent navigation (Blum and Abbott, 1996) and would be consistent with recent observations of asymmetric place field changes during experience (Mehta et al., 2000) and with the observation that CA1 pyramidal cells can encode temporal information as well as an animal's current location (Frank et al., 2000).

\section{Experience-dependent reductions in the magnitude of spike amplitude attenuation}

In the present study, we suggest that the longer timescale experience-dependent changes in the magnitude of amplitude attenuation within a burst may serve as an indicator of synaptic or cellular plasticity. Systematic changes in the kinetics of a cell's voltage-gated ion channels during environmental exposure would be expected to have a number of functional consequences for information processing within hippocampal pyramidal cells. First, because of reductions in attenuation, action potentials that initially failed to enter the distal dendrites of a cell would have an increased efficiency of back-propagation, and the ability to strengthen inputs onto distal dendrites would be enhanced. Second, because voltage-gated ion channels influence the integration of EPSPs within a cell, a biophysical change that alters the kinetics of sodium and potassium channels will also alter the filtering and integration of EPSPs within a cell's dendrites. In particular, a reduction in potassium activation and/or a reduction in sodium channel inactivation will not only reduce attenuations in spike amplitude but will also boost the effectiveness with which dendritic EPSPs propagate to a pyramidal cell's soma (Johnston et al., 1996; Hoffman et al., 1997; Sourdet and Debanne, 1999). Thus, an experience-dependent reduction in amplitude attenua- 
tion may reflect a biophysical change that allows electrical impulses to propagate more effectively within hippocampal pyramidal cells in both retrograde and anterograde directions. Such an increase in cellular responsiveness may allow the cell to respond both sooner and more robustly to subsequent input trains, a prediction that is consistent with the observation that the firing rate of a hippocampal pyramidal cell increases with experience and the first spike in a field also comes earlier with experience (Mehta et al., 2000).

\section{REFERENCES}

Andersen P, Moser EI (1995) Brain temperature and hippocampal function. Hippocampus 5:491-498.

Bear MF, Malenka RC (1994) Synaptic plasticity: LTP and LTD. Curr Opin Neurobiol 4:389-399.

Blum KI, Abbott LF (1996) A model of spatial map formation in hippocampus of the rat. Neural Comput 8:85-93.

Buzsaki G, Penttonen M, Nadasy Z, Bragin A (1996) Pattern and inhibition-dependent invasion of pyramidal cell dendrites by fast spikes in the hippocampus in vivo. Proc Natl Acad Sci USA 93:9921-9925.

Colbert CM, Magee JC, Hoffman D, Johnston D (1997) Slow recovery from inactivation of $\mathrm{Na}+$ channels underlies the activity-dependent attenuation of dendritic action potentials in hippocampal CA1 pyramidal neurons. J Neurosci 17:6512-6521.

Csicsvari J, Hirase H, Czurko A, Buzsaki G (1998) Reliability and state dependence of pyramidal cell-interneuron synapses in the hippocampus: an ensemble approach in the behaving rat. Neuron 21:179-189.

Csicsvari J, Hirase H, Czurko A, Mamiya A, Buzsaki G (1999) Oscillatory coupling of hippocampal pyramidal cells and interneurons in the behaving rat. J Neurosci 19:274-287.

Frank L, Brown E, Wilson MA (2000) Trajectory encoding in the hippocampus and entorhinal cortex. Neuron 27:169-178.

Giovannini MG, Giovannelli L, Bianchi L, Kalfin R, Pepeu G (1997) Glutamatergic modulation of cortical acetylcholine release in the rat: a combined in vivo microdialysis, retrograde tracing, and immunohistochemical study. Eur J Neurosci 9:1678-1689.

Gray CM, Maldonado PE, Wilson MA, McNaughton B (1995) Tetrodes markedly improve the reliability and yield of multiple single unit isolation from multi-unit recordings in cat striate cortex. J Neurosci Methods 61:43-54.

Grunze HC, Rainnie DG, Hasselmo ME, Barkai E, Hearne EF, McCarley RW, Greene RW (1996) NMDA-dependent modulation of CA1 local-circuit inhibition. J Neurosci 16:2034-2043.

Helmchen F, Svoboda K, Denk W, Tank DW (1999) In vivo dendritic calcium dynamics in deep-layer cortical pyramidal neurons. Nat Neurosci 2:989-996.

Henze DA, Borhegyi Z, Csicsvari J, Mamiya A, Harriz KD, Buzsaki G (2000) Intracellular features predicted by extracellular recordings in the hippocampus in vivo. J Neurophysiol 84:390-400.

Hoffman DA, Johnston D (1998) Downregulation of transient K+ channels in dendrites of hippocampal CA1 pyramidal neurons by activation of PKA and PKC. J Neurosci 18:3521-3528.

Hoffman DA, Johnston D (1999) Neuromodulation of dendritic action potentials. J Neurophysiol 81:408-411.

Hoffman DA, Magee JC, Colbert CM, Johnston D (1997) K+ channel regulation of signal propagation in dendrites of hippocampal pyramidal neurons. Nature 387:869-875.

Jaffe D, Johnston D, Lasser-Ross N, Lisman JE, Miyakawa H, Ross RN
(1992) The spread of NA+ spikes determines the pattern of dendritic $\mathrm{Ca}^{2+}$ entry into hippocampal neurons. Nature 357:244-246.

Johnston D, Magee JG, Colbert CM, Christie BR (1996) Active properties of neuronal dendrites. Annu Rev Neurosci 19:165-186.

Jung HY, Mickus T, Spruston N (1997) Prolonged sodium channel inactivation contributes to dendritic action potential attenuation in hippocampal pyramidal neurons. J Neurosci 17:6639-6646.

Kentros C, Hargreaves E, Hawkinsm RD, Kandel ER, Shapiro M, Muller RV (1998) Abolition of long-term stability of new hippocampal place cell maps by NMDA receptor blockade. Science 280:2121-2126.

Magee JC, Johnston D (1995) Synaptic activation of voltage-gated channels in the dendrites of hippocampal pyramidal neurons. Science 268:301-304.

Magee JC, Johnston D (1997) A synaptically controlled, associative signal for Hebbian plasticity in hippocampal neurons. Science 275:209-213.

McHugh TJ, Blum KI, Tsien JZ, Tonegawa S, Wilson MA (1996) Impaired hippocampal representation of space in CA1-specific NMDAR1 knockout mice. Cell 87:1339-1349.

Mehta MR, Barnes CA, McNaughton BL (1997) Experience dependent asymmetric expansion of hippocampal place fields. Proc Natl Acad Sci USA 94:8918-8921.

Mehta MR, Quirk MC, Wilson MA (2000) Experience dependent asymmetric shape of hippocampal receptive fields. Neuron 25:707-715.

Morris RG, Anderson E, Lynch GS, Baundry M (1986) Selective impairment of learning and blockade of long-term potentiation by a $N$-methyl-D-aspartate receptor antagonist, AP5. Nature 319:774-776.

O'Keefe J, Dostrovsky J (1971) The hippocampus as a spatial map: preliminary evidence from unit activity in the freely moving rat. Brain Res 34:171-175.

O'Keefe J, Nadel L (1978) The hippocampus as a cognitive map. London: Oxford UP.

Quirk MC, Wilson MA (1999) Interaction between spike waveform classification and temporal sequence detection. J Neurosci Methods 94:41-52.

Ranck Jr JB (1973) Studies of single neurons in the dorsal hippocampal formation and septum in unrestrained rats. I. Behavioral correlates and firing repertoires. Exp Neurol 41:461-531.

Roberson ED, English JD, Sweatt JD (1996) A biochemist's view of long-term potentiation. Learn Mem 3:1-24.

Sarter M, Bruno JP (1997) Cognitive functions of cortical acetylcholine: toward a unifying hypothesis. Brain Res Rev 23:28-46.

Sourdet V, Debanne D (1999) The role of dendritic filtering in associative long-term synaptic plasticity. Learn Mem 6:422-447.

Spruston N, Schiller Y, Stuart G, Sakmann B (1995) Activity-dependent action potential invasion and calcium influx into hippocampal CA1 dendrites. Science 268:297-300.

Tsubokawa H, Ross RN (1996) IPSPs modulate spike backpropagation and associative $\left[\mathrm{Ca}^{2+}\right] \mathrm{I}$ changes in the dendrites of hippocampal CA1 pyramidal neurons. J Neurophysiol 76:2896-2906.

Tsubokawa H, Ross RN (1997) Muscarinic modulation of spike backpropagation in the apical dendrites of hippocampal CA1 pyramidal neurons. J Neurosci 17:5782-5791.

Tsubokawa H, Offermanns S, Simon M, Kano M (2000) Calciumdependent persistent facilitation of spike backpropagation in the CA1 pyramidal neurons. J Neurosci 20:4878-4884.

Wilson MA, McNaughton BL (1993) Dynamics of the hippocampal ensemble code for space. Science 261:1055-1058.

Yang SN, Tang YG, Zuker RS (1999) Selective induction of LTP and LTD by postsynaptic [CA $\left.{ }^{2+}\right]$ I elevation. J Neurophysiol 81:781-787. 